\title{
Does targeting key-containers effectively reduce Aedes aegypti population density?
}

\author{
Rafael Maciel-de-Freitas and Ricardo Lourenço-de-Oliveira \\ Laboratório de Transmissores de Hematozoários, Instituto Oswaldo Cruz, Rio de Janeiro, Brazil
}

Summary

овJеCtives The elimination of Aedes aegypti breeding sites has been broadly adopted worldwide to keep vector population density below a critical threshold. We observed the effectiveness of targeting the most productive containers on adult $A$. aegypti females density, which was evaluated weekly. METHODS AND RESULTS Adult mosquitoes were collected weekly over 55 weeks and pupal surveys were done in intervals of 4 months to determine container productivity and guidelines for interventions. Pupal surveys indicated that water tanks ( $72 \%$ of pupae in first survey) and metal drums (30.7\% of pupae in second survey) were the most productive container types. We observed a dramatic but short-term decrease in weekly adult female A. aegypti density after covering 733 water tanks with nylon net. A long-term decrease in female adult population density was achieved only when we covered both water tanks and metal drums. Overall, pupae abundance and pupae standing crop diminished after netting water tanks and metal drums. Pupae per person, per hectare and per house decreased gradually between the first and the third pupal surveys, suggesting that targeting the most productive container types (water tanks and metal drums) produced a reduction in adult population density and infestation levels. CONCLUSION Overall, targeting the most productive container types caused the adult mosquito density to decrease over time, supporting the assumption that this intervention is an effective tool for dengue control. However, this effect was observed only when both water tanks and metal drums were covered, possibly due to the functional similarity between these container types, which are large, often shaded, perennial water storage containers.

keywords dengue, control, container, disease transmission, diptera

\section{Introduction}

The World Health Organization (WHO) estimates that 50 million cases of dengue fever occur annually, with more than 2.5 billion people living at risk, mainly in tropical regions (Nathan \& Dayal-Drager 2007). Since there are no antiviral drugs and vaccines available for commercialisation, vector control is the primary means to reduce dengue transmission. The main vector of dengue worldwide is the mosquito Aedes aegypti, which is well adapted to urbanised areas especially the surroundings of human dwellings and breeds often in man-made containers (Braks et al. 2003; Maciel-de-Freitas et al. 2007a; David et al. 2009).

The final outcome of every vector control campaign is to reduce $A$. aegypti adult population density below a threshold level where mosquitoes are no longer of public health importance (Focks 2003). However, control activities in many dengue endemic metropolitan cities have focused on reducing mosquito larvae and pupae without measuring the impact of control activities on adult populations. In Brazil, as well as in other dengue endemic countries, the limited number of vector control workers prevents frequent inspections of all households. Routine vector control surveys occur four to six times per year in a random sample of households in 146 sentinel cities in Brazil (Coelho et al. 2008). Therefore, there is the need to identify more efficient, alternative vector control strategies that have a measurable impact on adult mosquito population densities.

Container types vary in epidemiological importance, since some containers produce more pupae than others (Focks et al. 1981; Tun-Lin et al. 1995). Traditional entomological indices, such as the House (HI) and Breteau indices (BI), measured larval populations. However, pupal surveys provide a more accurate proxy estimate of adult population density resulting in more useful infestation indices, such as pupae per person (PPP) and pupae per hectare (PPH) (Connor \& Monroe 1923; Breteau 1954; Focks \& Chadee 1997; Focks et al. 2000; Morrison et al. 2004; Schneider et al. 2004; Barrera et al. 2006).

The main objective of targeting the most productive container types in a given area is to optimise labour 
R. Maciel-de-Freitas \& R. Lourenço-de-Oliveira Reducing A. aegypti density

efficiency and minimise costs while maximizing vector population reduction (Focks \& Chadee 1997; Focks et al. 2000; Focks 2003). Despite the logic underlying this theory, few studies have assessed the effect of targeting and eliminating the most productive container types on adult mosquito population densities (Scott \& Morrison 2003; Tun-Lin et al. 2009). Hence, in this study we evaluated the impact of eliminate the most productive container types on A. aegypti population density in a dengue endemic urban centre in Brazil.

\section{Materials and methods}

\section{Study area}

The study was performed in an urban settlement of Rio de Janeiro named Tubiacanga, an isolated, low to middle class suburban area that is relatively homogeneous and well planned. Most homes have two to three bedrooms, large yards with moderate vegetation cover, and streets are unpaved with well-maintained sidewalks. The area has poor basic sanitation, with irregular piped water distribution and garbage collection. Tubiacanga is located in a lowland coastal area, surrounded on two sides by Guanabara Bay, which is likely to have restricted mosquito immigration and emigration. The settlement comprises 2900 people living in 867 households, with a population density of 337.4 hab/ha, distributed across 14 blocks.

\section{Dengue incidence and climatic variables}

From 2000 to 2009, the Municipality of Rio de Janeiro had 339338 registered cases of dengue fever. During this 10year period, Rio de Janeiro suffered two dengue outbreaks due to the re-emergence of serotype 3 in 2002 and serotype 2 in 2008. Dengue in Rio de Janeiro is strongly seasonal, with around $85 \%$ of cases reported in the rainy summer, between January and April (Figure 1). The higher precipitation and mean temperature in summer increases the number of oviposition sites and accelerates immature development time, producing higher standing crop during this season. In Tubiacanga mean total monthly precipitation from January to April is $636.2 \mathrm{~mm}$ and mean daily temperature during this period is $26.25^{\circ} \mathrm{C}$ (Figure 2).

\section{Monitoring adult $A$. aegypti population}

From July 2008 to August 2009 (55 weeks), we monitored weekly the adult A. aegypti population density. Adults were collected in 40 randomly selected houses, representing approximately $5 \%$ of the homes in the study area. Each week 20 households were sampled using backpack aspirators (Clark 1994) and 20 were sampled using MosquiTRAPs (Fávaro et al. 2006). The backpack aspirator is the most effective collection method for adult A. aegypti, since it captures males and females at any physiological status, allowing more accurate adult density estimates (Scott \& Morrison 2003). Aspiration was conducted inside the household and in the peridomestic environments for 15$20 \mathrm{~min}$ per house. The MosquiTRAP was homogeneously installed in the study area, with approximately 1 trap installed in one household per block, and the traps remained in the same households during the entire study period. MosquiTRAP consisted of a matte-black container with around $300 \mathrm{ml}$ of water and a black adhesive card in its interior to trap gravid A. aegypti females. AtrAedes, a commercial insect attractant for gravid females, was glued to the adhesive card (Fávaro et al. 2006). Water from traps was changed weekly and the adhesive cards were examined weekly for mosquitoes and were replaced bimonthly, as recommended by the manufacturer.

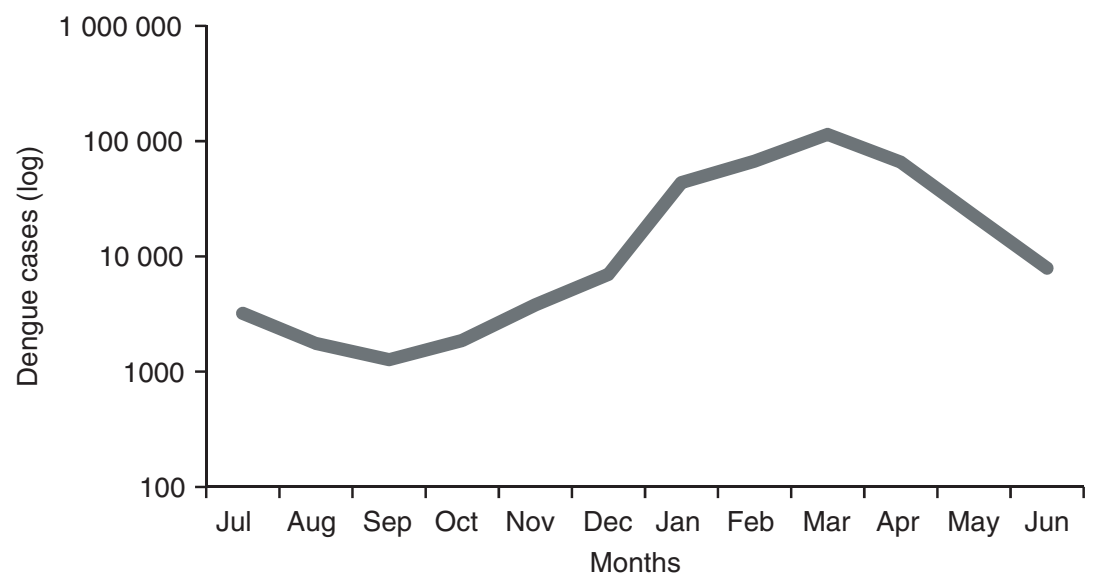

Figure I Dengue incidence in Rio de Janeiro city during 2000-2009. The increase of temperature and precipitation in the summer season (December-March) produces a higher standing crop of Aedes aegypti, which consequently influences dengue transmission. Dengue cases on $y$-axis are $\log$ scaled. 
R. Maciel-de-Freitas \& R. Lourenço-de-Oliveira Reducing A. aegypti density

Figure 2 Rainfall ( $\mathrm{mm})$ and mean temperature observed in Tubiacanga between July 2008 and July 2009.

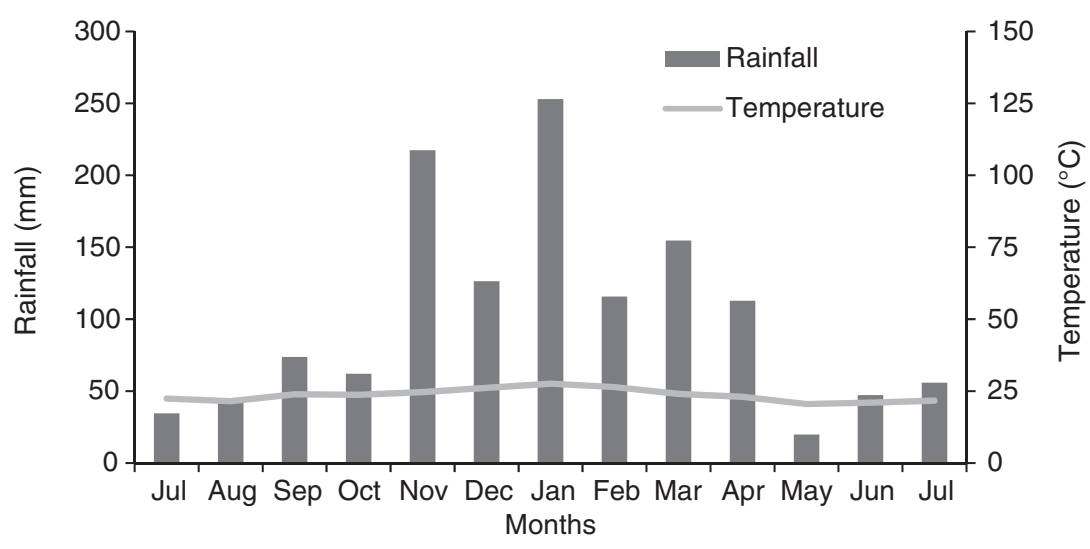

To evaluate the epidemiological relevance of each container type per survey in Tubiacanga, a set of container characteristics was estimated. Container abundance $(\mathrm{CoAb})$ and pupal abundance $(\mathrm{PAb})$ was the absolute number of water-storage container and pupae per survey, respectively, and proportion of pupae ( $\mathrm{PoP})$ was calculated for each container type. The frequency of pupae positive (FPP) was calculated as the number of pupae positive container/number of all inspected containers. Pupae standing crop was estimated as proposed by Focks et al. (2000), as the product of the number of pupae present, the proportion of pupae that are females (0.50), and the rate of successful emergence (0.83) (values observed in Focks et al. 1993). Pupae per person (PPP $=$ number of pupae/number of people in inspected houses) and pupae per hectare $(\mathrm{PPH}=$ number of pupae/inspected ha) were also evaluated for each survey.

\section{Statistical analysis}

To test the short-term impact of interventions on adult mosquito population density, we performed a two-sample paired $t$ test comparing the average number of adults collected in the week of the intervention and the three previous weeks against the 4 weeks after intervention. To test if infestation levels significantly varied due to interventions (i.e. between surveys) we compared the mean of $\mathrm{HI}$ and PPP per block by a two-sample paired $t$ test (first $v s$. second surveys and second $v$ s. third surveys).

According to the Bartlett test, the number of immature per container $(P<0.0001)$ or per house $(P<0.001)$ was not normally distributed. Thus, to test if the interventions produced any shift in the overall pattern of container production, we compared the median number of pupae per container (independent of container type) by a two-bytwo Mann-Whitney test (first $v$ s. second surveys and 
second $v s$. third surveys). Statistical analyses were conducted using the software GraphPad Instat 3.00.

\section{Ethical considerations}

According to Fiocruz Ethical Committee, there is no need of ethical clearance to visit and inspect houses to search $A$. aegypti immature or adult forms, since households are informed about the project's aims and methodology, which did not request personal information or put households at risk. Only after receiving oral permission from the head of the household, public health agents entered the home to perform the research activities, including installation of MosquiTRAPs to be monitored weekly and reviewing containers during pupal surveys.

\section{Results}

During the three surveys, we conducted a total of 1762 household inspections in Tubiacanga, and collected 4602 larvae and 881 pupae (Table 1). Immature of A. albopictus, Ochlerotatus fluviatilis, Cx quinquefasciatus and Wyeomyia sp. were also collected, but results will focus on A. aegypti findings. A total of 2644 A. aegypti females were collected throughout the 55 weeks of the experiment, 2007 (75.9\%) of them with MosquiTRAPs and 637 (24.1\%) with backpack aspirator.

Before the first intervention, between the first and ninth weeks, Tubiacanga had a mean \pm SD of $55.5 \pm 16.78$ females collected per week. In the first survey, we collected a total of 2341 larvae and 402 pupae of A. aegypti in 58 positive premises, resulting in a HI of 8.88 and a PPP of 0.1845 (Table 1). Of the 402 pupae collected, 291 (72.4\%) were captured in water tanks (Table 2 and Figure 3). Metal drums, which are often used to store water, presented low productivity, with just $3.48 \%$ of all pupae at that time. At this point, $7.12 \%$ of water tanks and $1.65 \%$ of metal drums were pupae positive (Table 2). PSC of

Table I Infestation levels observed in Tubiacanga during each pupal survey

\begin{tabular}{llll}
\hline & First survey & Second survey & Third survey \\
\hline $\begin{array}{l}\text { Houses inspected (\%) } \\
\text { Positive houses (\%) }\end{array}$ & $653(76.2)$ & $594(68.51)$ & $515(59.4)$ \\
$\begin{array}{l}\text { Breteau index } \\
\text { Persons in sampled } \\
\text { houses }\end{array}$ & 11.02 & $28(4.71)$ & $54(10.48)$ \\
$\begin{array}{l}\text { Pupae per person } \\
\text { Inspected hectare }\end{array}$ & 0.1845 & 1981 & 12.23 \\
$\begin{array}{l}\text { Pupae per hectare } \\
\text { Pupae per house }\end{array}$ & 6.26 & 0.1413 & 0.115 \\
\hline
\end{tabular}

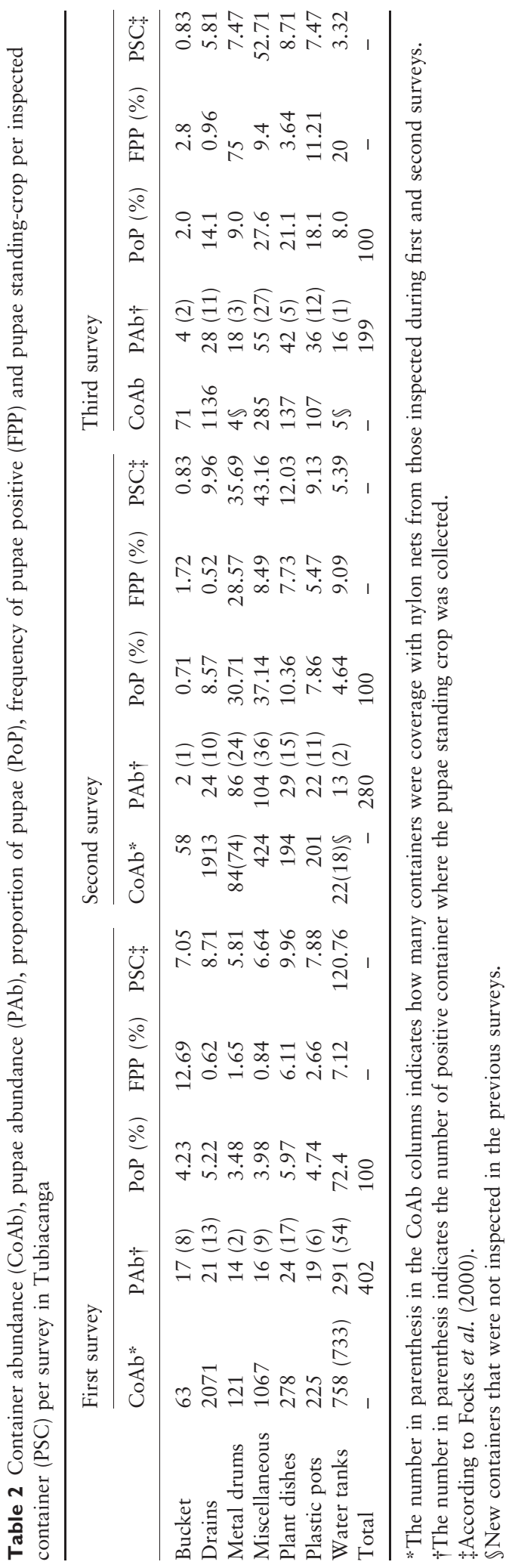


R. Maciel-de-Freitas \& R. Lourenço-de-Oliveira Reducing A. aegypti density

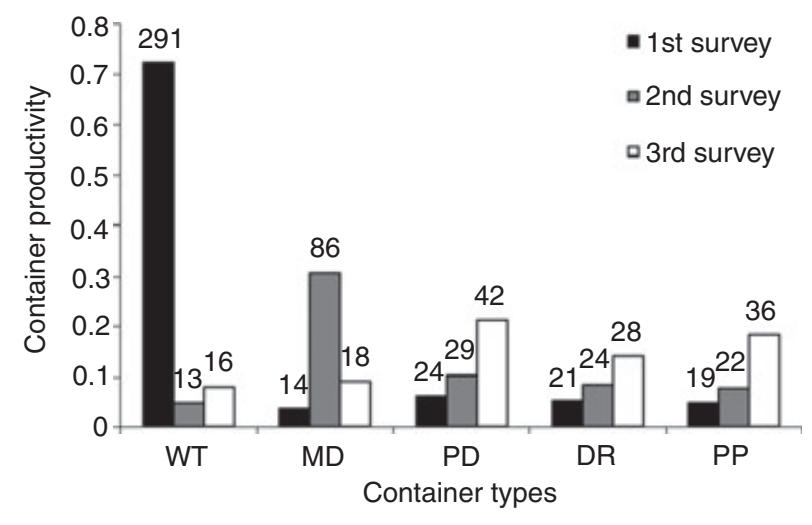

Figure 3 Container productive observed for Tubiacanga during each survey. Container abbreviation as follow: $\mathrm{BC}=$ bucket, $\mathrm{DR}=$ drain, $\mathrm{MD}=$ metal drums, $\mathrm{MI}=$ miscellaneous, $\mathrm{PD}=$ plant dish, PP = plastic pot, WT = water tank. Numbers above the bars indicate the absolute number of pupae collected in each container type.

water tanks were the highest of all positive containers, reaching a value of 120.76 , while metal drums and plant dishes were 5.81 and 9.96 , respectively. Other containers such as domestic drains, pots and plant dishes were also low productive with $5 \%$ of all pupae each. Therefore, after the first pupal survey and observing that water tanks were the most productive container type, we covered 733 water tanks with nylon net. We estimated that at least $96 \%$ remained covered until the end of the experiment 10 months later.

After netting 733 water tanks in the first intervention, we observed a rapid, intense, and significant decrease in adult mosquito population density $(t=3.866, \mathrm{df}=4$, $P=0.018)$, when $28.5 \pm 8.34$ females were collected between 10th and 13th weeks. However, this reduction in weekly adult density lasted only a few weeks after the intervention; by week 13 the adult population had returned to levels similar to those observed before the intervention (Figure 4). The mean \pm sd mosquitoes captured between the 14th and 25 th week was $60.58 \pm 17.78$ females per week.

In the second survey (week 25) we collected 1413 larvae and 280 pupae of A. aegypti. Based on the HI per block, infestation levels decreased significantly 3 months after covering 733 water tanks $(t=4.652, \mathrm{df}=13, P=0.0005)$, suggesting that targeting water tanks was an effective intervention. However, the infestation level remained statistically similar if we assessed PPP per block $(t=1.617$, $\mathrm{df}=13, P=0.1299$ ). Surprisingly, we observed a dramatic increase in almost all container type productivities. Metal drums increased from $3.48 \%$ of pupae in the first survey to $30.71 \%$ of pupae in the second survey, with an almost ninefold increase in the number of pupae collected between surveys in this container type. The frequency of pupae positive metal drums arose from $1.65 \%$ in the first survey to $28.57 \%$ in the second survey and PSC increased from 5.81 to 35.69 (Table 2). On the other hand, the number of pupae collected in water tanks fell from 291 in the first survey to 13 in the second survey (PSC from 120.76 to 5.39), found in two water tanks that were not covered in the first survey due to refusal of a household to participate in the intervention. Drains, pots and plant dishes also presented a higher productivity, frequency of pupae positive and PSC in the second rather in the first survey (Figure 3, Table 2). At that moment, we covered 74 metal drums, which remained covered up to the end of field trials.

In the 4 weeks after the second intervention, we collected $34.5 \pm 8.66$ females. The short-term reduction in weekly females collection was statistically significant, as it was after the first intervention $(t=3.497, \mathrm{df}=4, P=0.025)$. The cumulative effect of netting water tanks and metal drums
Figure 4 The total number of Aedes aegypti females collected each week by backpack aspirator and MosquiTRAP in Tubiacanga. Arrows indicate when we carried out the interventions.

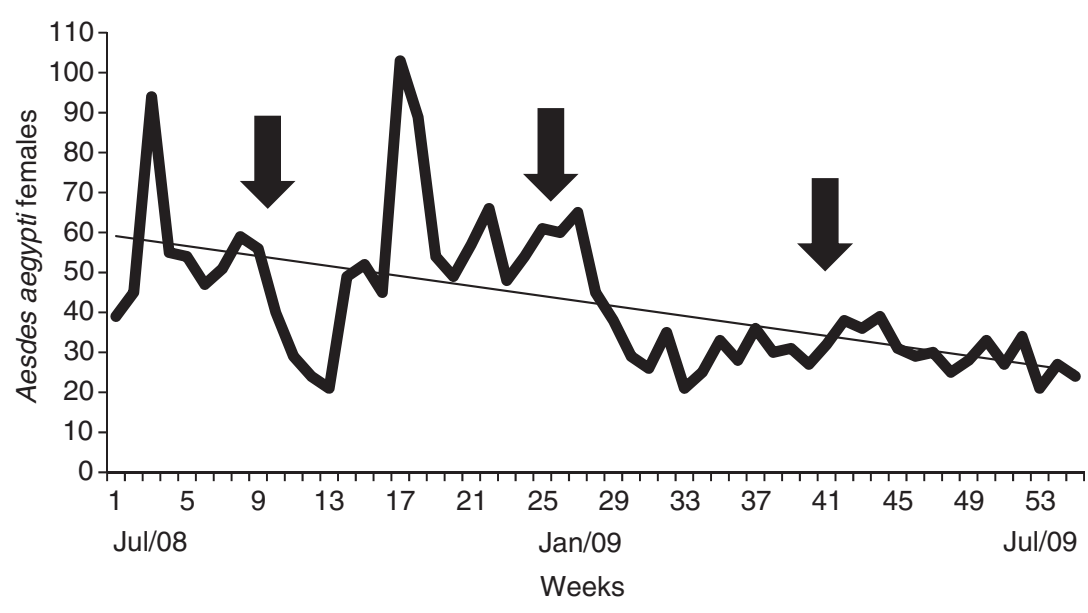


R. Maciel-de-Freitas \& R. Lourenço-de-Oliveira Reducing A. aegypti density

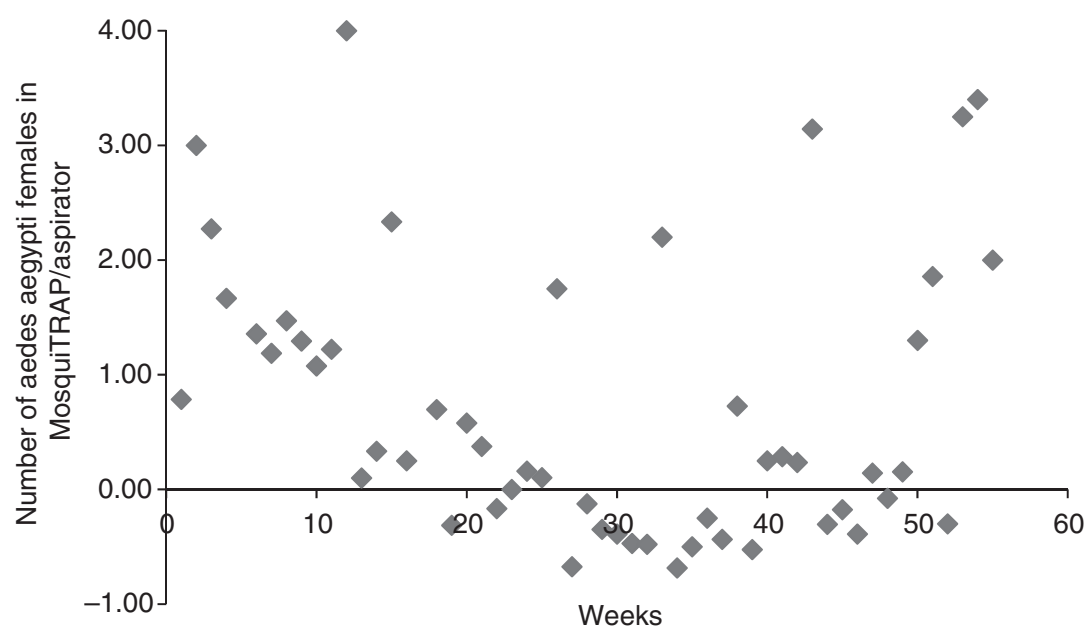

Figure 5 Compared efficiency of MosquiTRAP and backpack aspirator during the study period. Points above the $x$-axis indicate higher efficiency of the MosquiTRAP; points below the $x$-axis indicate higher efficiency of the backpack aspirator. produced a long-term reduction in female mosquito density for the remainder of the study (mean \pm SD was $30.11 \pm 4.98$ ) (Figure 4). Overall, the adult mosquito density presented a tendency to decrease in time in Tubiacanga, supporting the assumption that targeting the most productive containers is an effective tool to reduce vector population density and thus dengue transmission.

Finally, in the third pupal survey, when all water tanks and metal drums were still unavailable for mosquito oviposition, we collected many fewer A. aegypti larvae and pupae (848 larvae and 199 pupae). Although the relative productivity in small containers increased between second and third surveys (drains from 8.5 to 14.1; plant dishes from 10.3 to 21.1 ; plastic pots from 7.8 to 18.1 ), the total pupae standing crop dropped (drains from 9.96 to 5.81; plant dishes from 12.03 to 8.71 ; plastic pots from 9.13 to 7.47 ). Metal drum productivity fell to $9 \%$. From the 18 pupae collected in metal drums during third survey, 10 were in two metal drums not inspected in the second survey and 8 were found in one metal drum with holes in the nylon net.

The use of HI per block or PPP per block to evaluate the efficiency of an intervention must be carefully analysed since the indices produced conflicting results. The infestation levels measured by $\mathrm{HI}$ was significantly higher in the third survey than in the second $(t=3.875, \mathrm{df}=13, P=0.0019)$, but statistically similar if analysed using PPP $(t=1.459$, $\mathrm{df}=13, P=0.168)$. The median number of $A$. aegypti pupae collected per container was similar during first and second surveys ( $U=642.5, P=0.793)$, but decreased significantly on the third survey $(U=333.5, P<0.001)$.

\section{Backpack aspirator and MosquiTRAP efficacy}

Overall, MosquiTRAP was more efficient than backpack aspirator in Tubiacanga, where it collected $75.9 \%$ of adult female A. Aegypti (Figure 5). A mean of 0.87 A. aegypti females was collected per MosquiTRAP/day, against 0.52 when using backpack aspirator.

\section{Discussion}

It has been traditionally assumed that the most costeffective means to prevent dengue transmission is through vector control. In this study we found that eliminating the most productive containers reduced the density of adult A. aegypti over the long term, thus probably leading to a reduction in mosquito-human contact rates and consequently on dengue transmission. The adoption of new approaches such as this to control the dengue vector, must be encouraged due to the difficulties faced by public health authorities in dengue endemic countries, such as insecticide resistance, rapid and uncontrolled urbanisation, ineffectively application of resources, inaccessibility of some areas to control agents due to urban violence, and continued application of ineffective control methods (Gubler 2002; Montella et al. 2007; Morrison et al. 2008; Eisen et al. 2009).

Water tanks were the most productive container type in Tubiacanga at the beginning of the study before any intervention. This finding agrees with previous studies that found that large containers used by households for water storage were often key-mosquito breeding containers in areas such as Tubiacanga, where piped water distribution is irregular (Barrera et al. 1993; Maciel-de-Freitas et al. 2007a). After covering water tanks, we observed a rapid, significant and dramatic decrease in weekly adult mosquito collection. Therefore, it is reasonable to assume that our intervention produced a considerable short-term impact on vector density. The adult female mosquito population remained at lower levels for only a few weeks after the first 
R. Maciel-de-Freitas \& R. Lourenço-de-Oliveira Reducing A. aegypti density

intervention and, remarkably, mean vector density per week returned to the levels observed prior to the intervention. This data suggests that eliminating only the most productive container type may be an effective but shortterm control tool. This also suggests that females were able to locate alternative breeding containers (i.e. metal drums) to re-establish their populations density 4-5 weeks. The median number of pupae collected in the first and second surveys was statistically similar, reinforcing the absence of a long-term effect on mosquito density due to targeting water tanks. Water tanks and metal drums are similar in functionality: they are large and often shaded water reservoirs. Besides, they are abundant and perennial containers, i.e. they are always available for mosquito oviposition year-round if not properly covered. Thus, despite the lower expected impact of covering 74 metal drums in comparison of netting 733 water tanks, a harsh and long-term impact on A. aegypti density was achieved only after covering both containers. The cumulative effect of eliminating water tanks and metal drums, containers that together can hold more than $70 \%$ of pupae in Tubiacanga irrespective of seasonality (Maciel-de-Freitas et al. 2007a), probably produced the long-term decrease in vector density observed after the second intervention. Besides, the reduction in adult density started in January/ 2009, a month with intense rainfall and high temperatures, a suitable ambient for $A$. aegypti mosquitoes (Honório et al. 2009). In a previous report, Honório et al. (2009) showed high week collection of adult A. aegypti in Tubiacanga was performed between October and February. Thus, we believe the dramatic decline in adult $A$. aegypti collection after first and second surveys, i.e. January/2009, may be explained by covering two container types that have functional similarities.

Small containers such as domestic drains, plastic pots and plant dishes presented a significant shift in their pupal productivity during field study. In the first survey, they presented a low epidemiological relevance due to their low pupal productivity. The PSC of small containers increased after the netting water tanks, however, when water tanks and metal drums were both netted, their PSC decreased to levels similar to those observed before the first intervention. The main explanation for the initial increase in the PSC of small containers may be the opportunistic behaviour and ecological plasticity of A. aegypti in relation to manmade larval sites. It has been shown that the eggs of A. aegypti females are laid in several containers in multiple batches, a behaviour known as 'skip oviposition' (Harrington \& Edman 2001; Colton et al. 2003). The decrease of the PSC of small containers after netting metal drums and water tanks reinforces the hypothesis that mosquito density was affected only when functionally similar large and productive containers were concomitantly targeted.

Traditional indices such as Breteau (BI) and House Index (HI) presented a different pattern than pupae per person (PPP) and pupae per hectare (PPH). While PSC and adult population density diminished between first and third survey, BI and $\mathrm{HI}$ increased, suggesting that different indexes must be calculated and considered to evaluate mosquito infestation and assess dengue transmission risk. Several previous reports showed PP and PPH provide more accurate and reliable infestation indices than $\mathrm{BI}$ and $\mathrm{HI}$ (Focks \& Chadee 1997; Focks et al. 2000; Morrison et al. 2004; Schneider et al. 2004; Barrera et al. 2006).

Our report produced a valuable opportunity to compare the efficiency of MosquiTRAP with backpack aspirator. Since MosquiTRAP is designed to capture gravid females and the backpack aspirator to collect resting male and female mosquitoes, comparisons between their efficiency must be carefully interpreted. Even though the MosquiTrap collects only gravid females, a higher number of A. aegypti insects were collected using this method. It is likely that removing water tanks and metal drums led to greater oviposition in MosquiTRAPs, thus increasing its efficiency relative to the backpack aspirator. However, the usefulness of MosquiTRAP in estimating seasonal variation in adult mosquito density and in assessing risk of dengue transmission still needs further evaluation.

Dengue control is labour-intensive and faces many challenges. Since no commercial vaccine or drug is commercially available to treat dengue-infected patients, the primary means to prevent disease transmission is vector control. In this study, we report a promising vector control strategy. We observed that netting the most productive containers produced a drastic and long-term reduction in adult mosquito density. We also observed that MosquiTRAP is more efficient than backpack aspirators in collecting adult female mosquitoes, a result that has been previously observed elsewhere (Fávaro et al. 2006). The adoption of new and effective tools for mosquito control, such as those reported in this study, and the elimination of unproductive strategies must be encouraged to support integrated vector management programs (Eisen et al. 2009; Lambrechts et al. 2009).

\section{Acknowledgements}

We would like to thank Mariana David, Anniely Ferreira, Reginaldo Rego, Roberto C. Peres, Renato Davi and Marcelo C. Santos for lab and field assistance. We also thank Dr Álvaro E. Eiras for allowing the use of MosquiTRAP, Apoio do Pronex-Rede Dengue, CNPq/ Faperj. 
R. Maciel-de-Freitas \& R. Lourenço-de-Oliveira Reducing A. aegypti density

\section{References}

Barrera R, Avila J \& Gonzalez ST (1993) Unreliable supply of potable water and elevated Aedes aegypti larval indexes - a causal relationship. Journal of the American Mosquito Control Association 9, 189-195.

Barrera R, Amador M \& Clark GG (2006) Use of the pupal survey technique for measuring Aedes aegypti (Diptera: Culicidae) productivity in Puerto Rico. American Journal of Tropical Medicine and Hygiene 74, 290-302.

Braks MAH, Honorio NA, Lourenço-de-Oliveira R, Juliano SA \& Lounibos LP (2003) Convergent habitat segregation of Aedes aegypti and Aedes albopictus (Diptera: Culicidae) in southeastern Brazil and Florida. Journal of Medical Entomology 40, 785-794.

Breteau H (1954) La fievre jaune en Afrique occidental française: un aspect de la medicine preventive massive. Bulletin of the World Health Organization 38, 720-724.

Clark GG (1994) Use of the CDC backpack aspirator for surveillance of Aedes aegypti in San Juan, Puerto Rico. Journal of the American Mosquito Control Association 10, 119-124.

Coelho GE, Burattini MN, Teixeira MG, Coutinho FAB \& Massad E (2008) Dynamics of the 2006/2007 dengue outbreak in Brazil. Memórias do Instituto Oswaldo Cruz 103, 535-539.

Colton YM, Chadee DD \& Severson DW (2003) Natural skip oviposition of the mosquito Aedes aegypti indicated by codominant genetic markers. Medical and Veterinary Entomology 17, 195-204.

Connor ME \& Monroe WM (1923) Stegomyia indices and their value in yellow fever control. American Journal of Tropical Medicine and Hygiene 3, 9-19.

Consoli RAGB \& Lourenço-de-Oliveira R (1994) Principais mosquitos de importância sanitária do Brasil. Fiocruz, Rio de Janeiro.

David MR, Lourenço-de-Oliveira R \& Maciel-de-Freitas R (2009) Container productivity, daily survival rates and dispersal of Aedes aegypti females in a high income dengue epidemic neighborhood of Rio de Janeiro: presumed influence of differential urban structure on mosquito biology. Memórias do Instituto Oswaldo Cruz 104, 927-932.

Eisen L, Beaty BJ, Morrison AC \& Scott TW (2009) Proactive vector control strategies and improved monitoring and evaluation practices for dengue prevention. Journal of Medical Entomology 46, 1245-1255.

Fávaro AE, Dibo MR, Mondini A et al. (2006) Physiological state of Aedes (Stegomyia) aegypti mosquitoes captured with MosquiTRAPs in Mirassol, São Paulo, Brazil. Journal of Vector Ecology 31, 285-291.

Focks DA (2003) A Review of Entomological Sampling Methods and Indicators for Dengue Vectors. World Health Organization, Geneva, 36 pp.

Focks DA \& Chadee DD (1997) Pupal survey: an epidemiologically significant surveillance method for Aedes aegypti: an example using data from Trinidad. American Journal of Tropical Medicine and Hygiene 56, 159-167.

Focks DA, Sackett SR, Bailey DL \& Dame DA (1981) Observations on container-breeding mosquitoes in New
Orleans, Louisiana, with an estimate of the population density of Aedes aegypti. American Journal of Tropical Medicine and Hygiene 30, 1329-1335.

Focks DA, Haile DG, Daniels E \& Mount GA (1993) Dynamic life table model for Aedes aegypti (Diptera: Culicidae) - analysis of the literature and model development. Journal of Medical Entomology 30, 1003-1017.

Focks DA, Brenner RA, Daniels E \& Hayes J (2000) Transmission threshold for dengue in terms of Aedes aegypti pupae per person with discussion of their utility in source reduction efforts. American Journal of Tropical Medicine and Hygiene 62, 11-18.

Gubler DJ (2002) The global emergence/resurgence of arboviral diseases as public health problems. Archives of Medical Research 33, 330-342.

Harrington LC \& Edman JD (2001) Indirect evidence against delayed "skip oviposition" behavior by Aedes aegypti (Diptera: Culicidae) in Thailand. Journal of Medical Entomology 38, 641-645.

Honório NA, Nogueira RMR, Codeço CT et al. (2009) Spatial evaluation and modeling of dengue seroprevalence and vector density in Rio de Janeiro, Brazil. PLoS Neglected Tropical Diseases 3, e545.

Lambrechts L, Knox TB, Wong J et al. (2009) Shifting priorities in vector biology to improve control of vector-borne disease. Tropical Medicine and International Health 14, 1505-1514.

Maciel-de-Freitas R, Marques WA, Peres RC, Cunha SP \& Lourenço-de-Oliveira R (2007) Variation in Aedes aegypti (Diptera: Culicidae) container productivity in a slum and a suburban district in Rio de Janeiro during dry and wet seasons. Memórias do Instituto Oswaldo Cruz 102, 489-496.

Montella IR, Martins AJ, Viana-Medeiros PF et al. (2007) Insecticide resistance mechanisms of Brazilian Aedes aegypti populations from 2001 to 2004. American Journal of Tropical Medicine and Hygiene 77, 467-477.

Morrison AC, Astete H, Chapilliquen F et al. (2004) Evaluation of a sampling methodology for rapid assessment of Aedes aegypti infestation levels in Iquitos, Peru. Journal of Medical Entomology 41, 502-510.

Morrison AC, Gutierrez EZ, Scott TW \& Rosenberg R (2008) Defining challenges and proposing solutions for control of the virus vector Aedes aegypti. PLoS Medicine 5, e68.

Nathan MB \& Dayal-Drager R (2007) Recent epidemiological trends, the global strategy and public health advances in dengue. In: WHO Scientific Working Group - Report on Dengue (ed WHO/TDR/SWG/08) World Health Organization, Geneva, pp. 30-34.

Schneider JR, Morrison AC, Astete H et al. (2004) Adult size and distribution of Aedes aegypti (Diptera: Culicidae) associated with larval habitats in Iquitos, Peru. Journal of Medical Entomology 41, 634-642.

Scott TW \& Morrison AC (2003). Aedes aegypti density and the risk of dengue-virus transmission. In: Ecological Aspects for Application of Genetically Modified Mosquitoes (eds W Takken \& TW Scott) FRONTIS, The Netherlands, pp. 187-206. 
R. Maciel-de-Freitas \& R. Lourenço-de-Oliveira Reducing A. aegypti density

Tun-Lin W, Kay BH \& Barnes A (1995) Understanding productivity, a key to Aedes aegypti mosquitoes surveillance. American Journal of Tropical Medicine and Hygiene 53, 595-601.

Tun-Lin W, Lenhart A, Nam VS et al. (2009) Reducing costs and operational constraints of dengue vector control by targeting productive breeding places: a multi-country non-inferiority cluster randomized trial. Tropical Medicine and International Health 14, 1143-1153.

Corresponding Author Rafael Maciel-de-Freitas, Laboratório de Transmissores de Hematozoários, Pavilhão Carlos Chagas, sala 414, $4^{\circ}$ andar, Instituto Oswaldo Cruz, Fiocruz, Rio de Janeiro, Brazil. E-mail: freitas@ioc.fiocruz.br 